\title{
GRADIENTE POSQUIRÚRGICO EN PACIENTES CON REEMPLAZO VALVULAR AÓRTICO Y SU ASOCIACIÓN CON EVENTOS CARDIOVASCULARES
}

\author{
POST-SURGICAL GRADIENT IN PATIENTS WITH AORTIC VALVE \\ REPLACEMENT AND ITS ASSOCIATION WITH CARDIOVASCULAR EVENTS
}

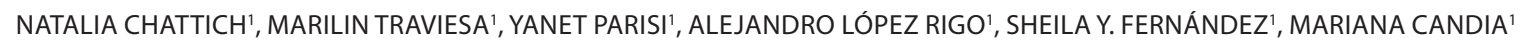

\section{RESUMEN}

Introducción. La cirugía de reemplazo valvular aórtico (CRVAo) constituye el tratamiento de elección en la estenosis aórtica (EAo) severa sintomática o con deterioro de la función ventricular. El gradiente posquirúrgico se ha visto asociado significativamente con la sobrevida y evolución a largo plazo. Sin embargo, su prevalencia y correlación con desenlaces clínicos en nuestro centro aún no ha sido estudiada formalmente.

Objetivo. Evaluar el gradiente posquirúrgico valvular en pacientes sometidos a CRVAo y su asociación con eventos cardiovasculares en el Instituto de Cardiología de Corrientes Juana F. Cabral.

Métodos. Estudio observacional, retrospectivo, unicéntrico. Se incluyeron pacientes sometidos a CRVAo por EAo severa y se evaluaron los gradientes valvular aórticos pre- y posquirúrgicos con un seguimiento a 6 meses.

Resultados. Se incluyeron 199 pacientes. El 86,9\% presentó hipertensión arterial, el $20,7 \%$ diabetes mellitus, 29,8\% dislipemia y el 10,1\% presentaba aorta bicúspide. Con respecto a los eventos cardiovasculares posquirúrgicos: 9,2\% presentó insuficiencia cardíaca, $1 \%$ bloqueo auriculoventricular completo, 1,5\% requirió implante de marcapasos definitivo, 2,1\% fibrilación auricular, 3,1\% derrame pericárdico severo y el 2,1\% endocarditis infecciosa, sin encontrarse asociación entre los eventos cardiovasculares posquirúrgicos de ambas ramas. La mortalidad posoperatoria fue del 2,5\%. Comparando las variables ecocardiográficas pre- y posquirúrgicas se observó disminución de los gradientes y aumento del área valvular en forma significativa. Se observó que el $37,1 \%$ de los pacientes operados presentó disminución respecto al valor basal pero persistió con gradientes postquirúrgicos elevados. En dichos pacientes, se observó que el gradiente prequirúrgico medio era $10 \mathrm{mmHg}$ mayor con respecto a los pacientes que luego de la cirugía presentaron gradientes normales.

Conclusión. La prevalencia de gradiente posquirúrgico elevado en nuestro centro es elevada. El gradiente posquirúrgico elevado no se asoció con aumento de eventos cardiovasculares registrados.

Palabras clave: estenosis de la válvula aórtica, cirugía cardíaca.

\section{ABSTRACT}

Introduction. Aortic valve replacement surgery (AVR) is the treatment of choice in severe aortic stenosis (AS) that is symptomatic or has deteriorated ventricular function. The postoperative gradient has been significantly associated with long term survival and evolution. However, its prevalence and correlation with clinical outcomes in our center has not yet been formally studied.

Objective. To evaluate the postoperative valvular gradient in patients undergoing AVR and its association with cardiovascular events in the Corrientes Institute of Cardiology, Juana F. Cabral.

Methods: Observational, retrospective single-center study. We included patients undergoing AVR due to severe AS, and the aortic valve gradients before and after surgery were evaluated during a 6-month follow-up.

Results. 199 patients were included. 86.9\% presented hypertension, 20.7\% diabetes mellitus, $29.8 \%$ dyslipidemia, and $10.1 \%$ had bicuspid aorta. With respect to postsurgical cardiovascular events: $9.2 \%$ presented heart failure, $1 \%$ complete atrioventricular block, $1.5 \%$ required permanent pacemaker implantation, $2.1 \%$ atrial fibrillation, 3.1\% severe pericardial effusion and $2.1 \%$ infective endocarditis, with no association between postsurgical cardiovascular events of both branches. The postoperative mortality was $2.5 \%$. Comparing the preoperative and post-surgical echocardiographic variables, a decrease in the gradients and a significant increase in the valve area were observed. It was observed that $37.1 \%$ of the operated patients presented decrease with respect to the baseline value but persisted with high postoperative gradients. In these patients, it was observed that the average presurgical gradient was $10 \mathrm{mmHg}$ higher than in patients who presented normal gradients after surgery.

Conclusion. The prevalence of a high postoperative gradient in our center is high. The elevated post-surgical gradient was not associated with an increase in recorded cardiovascular events.

Keywords: aortic stenosis, cardiac surgery.

REVISTA CONAREC 2018;33(147):305-307 | DOI:10.32407/RCON/2018147/0305-0307

\section{INTRODUCCIÓN}

La estenosis aórtica constituye una de las afectaciones valvulares cardíacas de mayor prevalencia a nivel mundial, que aumenta de manera directamente proporcional con la edad ${ }^{1}$

No existen dudas respecto del beneficio que otorga la cirugía de reemplazo valvular en lo referente al curso evolutivo y pronóstico de los pacientes afectados por esta valvulopatía. A pesar de esto, la sobrevida alejada, sobre todo de los pacientes jóvenes sometidos a dicho proce-

1. Residente de Cardiología

Instituto de Cardiología de Corrientes Juana F. Cabral

$\triangle$ Correspondencia: Dra. Natalia Chattich. Bolivar 1334, Corrientes, provincia de Corrientes, Rep. Argentina. natichattich@hotmail.com

Los autores declaran no tener conflictos de intereses.

Recibido: 10/12/2018| Aceptado: 15/12/2018 dimiento, es inferior a la de la población general. Si bien esto puede responder en parte al tipo de prótesis valvular elegida, está fundamentalmente ligado a las características particulares de cada paciente².

La mortalidad hospitalaria del procedimiento está relacionada con las características basales de la población y con la necesidad o no de realizar otro tipo de intervención asociada3.

La sobrevida y la evolución a largo plazo se encuentran determinadas

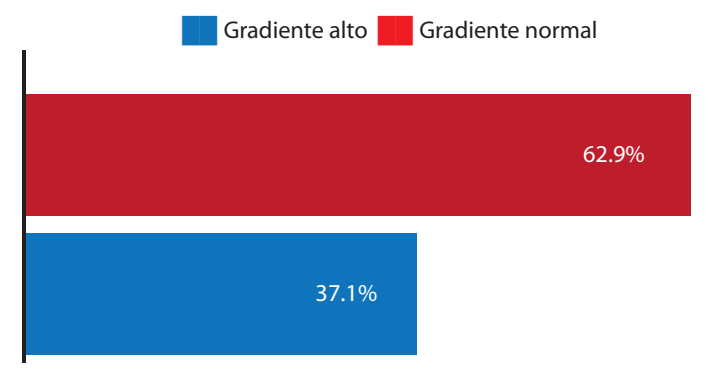

Figura 1. Porcentaje de pacientes que presentaron gradiente normal y gradiente alto en el posquirúrgico. 
Gradiente valvular alto $\square$ Gradiente valvular normal

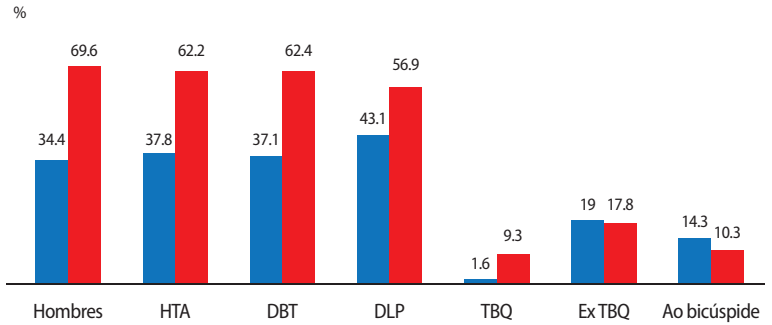

Figura 2. Características basales de los pacientes teniendo en cuenta el gradiente valvula post-quirúrgico normal y alto. G: gradiente. HTA: hipertensión arterial. DBT: diabetes mellitus. DLP: dislipemia. TBQ: tabaquismo. EX TBQ: extabaquismo. Ao: aorta.

por la edad, la función ventricular previa, la existencia o no de otras comorbilidades y el tipo de sustituto valvular, que también tendrá influencia en términos de clase funcional y calidad de vida ${ }^{4,5}$.

Con respecto al tipo de prótesis seleccionada y sus complicaciones, las mecánicas presentan mayor durabilidad pero son más propensas a sufrir complicaciones tromboembólicas debido al mismo material, por lo que requieren anticoagulación crónica con los riesgos propios de esta terapéutica. Los implantes biológicos se comportan en forma opuesta: habitualmente no requieren anticoagulación pero suelen sufrir procesos degenerativos más temprano.

El gradiente posquirúrgico elevado (definido como una velocidad transvalvular mayor a $3 \mathrm{~m} / \mathrm{s}$ según las últimas guías internacionales) se ha asociado significativamente en forma negativa en la sobrevida y evolución a largo plazo ${ }^{6}$.

Es por esto que posterior al reemplazo valvular aórtico (RVA) los pacientes deben ser evaluados en forma periódica incluyendo examen físico, radiografía de tórax, electrocardiograma, análisis de laboratorio y ecocardiograma a fin de determinar la aparición de complicaciones clínicas y relacionadas con la prótesis, tales como insuficiencia cardiaca, disfunción valvular, arritmias o endocarditis infecciosa $a^{7,8}$.

El objetivo de este trabajo es evaluar la prevalencia de gradientes valvulares posquirúrgicos aumentados en pacientes sometidos a cirugía de reemplazo valvular aórtico y su asociación con eventos cardiovasculares en nuestro centro.

\section{MATERIALES Y MÉTODOS}

Estudio observacional, retrospectivo, unicéntrico. Se incluyeron pacientes sometidos a CRVAo por EAo severa según las clasificaciones clínicas y ecocardiográficas vigentes, en el período comprendido desde enero del año 2013 hasta enero del año 2016. Los datos fueron recolectados de historias clínicas y de la base de datos del Servicio de Cirugía Cardiovascular del Instituto de Cardiología "Juana F. Cabral". Para determinar la diferencia entre gradientes pre- y posquirúrgico se tomaron en cuenta los ecocardiogramas pre- y posquirúrgicos de los pacientes. El seguimiento a los 6 meses luego de la intervención quirúrgica se realizó a través de la recolección de datos de historias clínicas en las que se confirmó internación por insuficiencia cardiaca, derrame pericárdico severo, endocarditis infecciosa, disfunción valvular o muerte. Se revisaron además datos demográficos como edad y sexo, factores

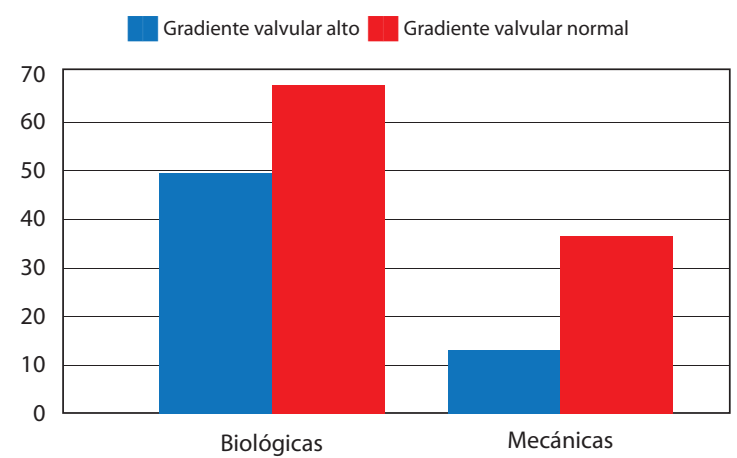

Figura 3. Comparación en cuanto al tipo de válvula y gradiente posquirúrgico normal o alto G. Valv: gradiente valvular

de riesgo cardiovascular como hipertensión arterial, diabetes mellitus, dislipidemia, tabaquismo y extabaquismo, así como la presencia o no de válvula aórtica bicúspide previa a la cirugía.

Los criterios de exclusión fueron los siguientes: pacientes sometidos a doble reemplazo valvular, pacientes con cirugía cardíaca previa, reemplazo valvular en contexto de disección aórtica con afección valvular y aquellos pacientes que no presentaran ecocardiograma realizado en el Instituto de Cardiología de Corrientes dentro de los 6 meses del posoperatorio.

\section{ANÁLISIS ESTADÍSTICO}

Las variables categóricas se expresaron mediante números y porcentajes, y las continuas, según su distribución, como media o mediana, con su correspondiente desvío estándar o intervalo intercuartílico. Para evaluar la asociación entre las variables continuas se utilizó el test de U de Mann-Whitney o test de T.Y para establecer asociación entre variables cualitativas se utilizó el test de Chi cuadrado y el análisis de riesgo asociado mediante el cálculo del odds ratio, con un intervalo de confianza del $95 \%$. El punto de corte utilizado para la significación estadística fue una $p<0,05$. Los datos recabados fueron registrados en una planilla de cálculo de Microsoft Excel. El análisis estadístico se realizó con el programa SPSS 21 para Windows.

\section{RESULTADOS}

Fueron incluidos 170 pacientes y excluidos 29. Del total de pacientes incluidos se observó que un 62,9\% presentó gradiente normal posquirúrgico y $37,1 \%$ gradiente aumentado, teniendo en cuenta un valor de velocidad transvalvular $>3 \mathrm{~m} / \mathrm{s}$ según las últimas recomendaciones de las guías de práctica clínica ${ }^{9}$ (Figura 1).

En cuanto a las características basales de los pacientes se observó que, del grupo con gradiente normal en el posquirúrgico, 63,6\% eran hombres, el 62,2\% tenía hipertensión arterial, el 62,4\% diabetes mellitus, 56,9\% dislipemia, 9,3\% tabaquismo, el 17,8\% extabaquismo y 10,3\% presentaban aorta bicúspide; mientras que dentro del grupo con gradiente alto $36,4 \%$ eran hombres, 37,8\% tenían hipertensión arterial, el 37,1\% diabetes mellitus, 43,1\% dislipemia, 1,6\% tabaquismo, 19\% extabaquismo y 14,3\% con aorta bicúspide (Figura 2).

Respecto al tipo de válvula, se observó que de aquellos con válvula biológica, 50 pacientes (42\%) presentaron gradiente alto y 69 (58\%) gradiente normal; de los pacientes con prótesis mecánica, 13 (26\%) presentaron gradiente aumentado y 38 (74\%) gradiente norma

(Figura 3). Comparando los valores de los pacientes con gradientes 


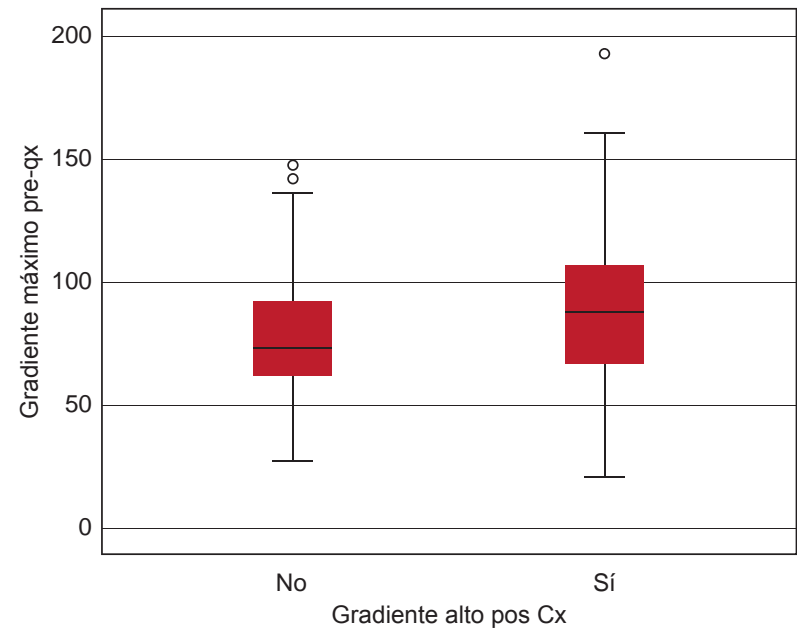

Figura 4. Comparación de medias de pacientes que presentaron gradientes normales vs. gradientes altos. Se observa que los pacientes que presentaron gradiente alto posquirúrgico presentaban gradientes prequirúrgicos más elevados. Gradiente máx pre-qx: gradiente máximo prequirúrgico. Gradiente alto pos $C x$ : gradiente alto posquirúrgico

postquirúrgicos normales, aquellos con gradientes aumentados presentaron una media de $10 \mathrm{mmHg}$ por encima del valor del primer grupo, con áreas valvulares más pequeñas. (Figura 4).

Finalmente no se registraron diferencias estadísticamente significativas con respecto a los eventos cardiovasculares posquirúrgicos comparando ambos grupos: se registró insuficiencia cardiaca en 9,5 vs. 11,3\% ( $p=0,464)$, bloqueo auriculoventricular completo 3,2 vs. $0 \%$ ( $p=0,138)$, requerimiento de marcapasos definitivo 4,8 vs. $0 \%(p=0,5)$, fibrilación auricular 3,2 vs. $1,9 \%(p=0,471)$, derrame pericárdico severo 4.9 vs. $2.9 \%$ ( $p=0,392)$, necesidad de pericardiocentesis 4,8 vs. 1,9\% ( $p=0,263)$, endocarditis 4,8 vs. $0,9 \%$ ( $p=0,147)$ y muerte 3,2 vs. $0,9 \%(p=0,309)$, respectivamente (Figura 5).

\section{DISCUSIÓN}

En este trabajo se observó que, en nuestro centro, un 37,1\% de los pacientes sometidos a CRVA persistió con gradiente posquirúrgico elevado sin que esto se haya asociado a una mayor tasa de eventos cardiovasculares.

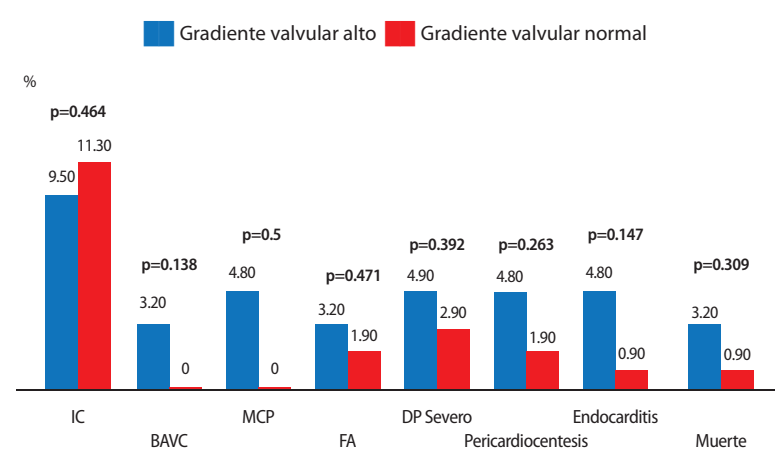

Figura 5. Eventos cardiovasculares postquirúrgicos registrados y su relación con gradientes normales o altos. No se encontraron diferencias significativas estadísticamente. IC: insuficiencia cardiaca. BAVC: bloqueo auriculoventricular completo. MCP: marcapasos. FA: fibrilación auricular. DP: derrame pericárdico.

Una de las limitaciones de nuestro trabajo consistió en no contar con el dato de la velocidad del tracto de salida del ventrículo izquierdo en los ecocardiogramas posquirúrgicos, con la cual se podría evaluar la disfunción valvular como una de las causas de dicho gradiente elevado.

Otro problema potencial a descartar en estos pacientes sería la discordancia prótesis-paciente (DPP), la cual se presenta cuando el área del orificio efectivo de una prótesis que funciona normalmente, es demasiado pequeña en relación al tamaño del cuerpo del paciente ${ }^{5,6}$. Algunos estudios han demostrado que la presencia de DPP afecta negativamente la reversión de la hipertrofia ventricular, la capacidad funcional e incluso la sobrevida en algunos subgrupos ${ }^{3,4}$.

Consideramos que estos datos pueden servir como base para futuros estudios y así ampliar los hallazgos encontrados.

\section{CONCLUSIÓN}

En este estudio se observó una elevada prevalencia de gradientes postquirúrgicos altos, sin esto asociarse significativamente con aumento de los eventos cardiovasculares registrados.

\section{BIBLIOGRAFÍA}

1. Lax J, Stutzbach P, Beck M, Perea FM, Cianciulli T, Grancelli H, et al. Consenso de Valvulopatías. Rev Argent Cardiol 2015;83(2).

2. Carmona-Ruiz HA, Arias-Godínez JA, Ávila-Vanzzini N, Romero-Aragonés C, Roldán-Gómez FJ, Ruiz-Esparza-Dueñas ME, et al. Evaluación postquirúrgica por ecocardiografía del paciente sometido a reemplazo valvular aórtico. ¿Se aplican las recomendaciones de las guías?. Rev Mex Cardiol 2014;25(3):158-62.

3. Vohra HA, Whistance RN, de Kerchove L, Glineur D, Noirhomme P, El Khoury G. Influence of higher valve gradient on long-term outcome after aortic valve repair. Ann Cardiothorac Surg 2013;2(1):30-9.

4. Koene BM, Soliman Hamad MA, Bouma W, Mariani MA, Peels KC, van Dantzig JM, et al. Can postoperative mean transprosthetic pressure gradient predict survival after aortic valve replacement?. Clin Res Cardiol 2014;103(2):133-40.

5. Nishimura RA, Otto CM, Bonow RO, Carabello BA, Erwin JP III, Guyton RA, et al. 2014 AHA/ACC Guideline for the Management of Patients With Valvular Heart Disease: executive summary: a report of the American College of Cardiology/American Heart Association Task Force on Practice Guidelines. Circulation 2014;129(23):2440-92.

6. Zoghbi WA, Chambers JB, Dumesnil JG, Foster E, Gottdiener JS, Grayburn PA, et al. Rec ommendations for evaluation of prosthetic valves with echocardiography and doppler ultrasound: a report From the American Society of Echocardiography's Guidelines and Standards Committee and the Task Force on Prosthetic Valves, developed in conjunction with the American College of Cardiology Cardiovascular Imaging Committee, Cardiac Imaging Committee of the American Heart Association, the European Association of Echocardiography, a registered branch of the European Society of Cardiology, the Japanese Society of Echocardiography and the Canadian Society of Echocardiography, endorsed by the American College of Cardiology Foundation, American Heart Association, European Association of Echocardiography, a registered branch of the European Society of Cardiology, the Japanese Society of Echocardiography, and Canadian Society of Echocardiography. J Am Soc Echocardiogr 2009;22(9):975-1014.

7. Bach DS. Echo/Doppler evaluation of hemodinamics after aortic valve replacement: principles of interrogation and evaluation of high gradients. JACC Cardiovasc Imaging 2010;3(3):296-304.

8. Del Corro I, Contreras A, Brenna E. ¿Es posible predecir gradientes transvalvulares elevados en la cirugía valvular aórtica? Rev Fed Arg Cardiol 2013;43(1)14-17. 